\title{
Ueber die in Jahre 1876 ausgeführte Längenbestimmung zwischen Berlin und Odessa.
}

Wenn ich nachstehend einige Angaben tiber die im Jahre 1876 ausgefuhrte telegraphische Längenbestimmung Berlin-Odessa zu machen mir erlaube, so veranlasst mich dazu einerseits der Umstand, dass bisher wenig oder nichts darüber bekannt geworden ist, andererseits die Befurchtung, dass eine ausführliche Publication uberhaupt nicht mehr erfolgen werde. Der genannte Anschluss der Odessaer Sternwarte an Berlin wurde in Erfüllung eines von dem damaligen Observator derselben Herrn Prof. Block wiederholt gegen Herrn Geheimrath Foerster geäusserten Wunsches und unter des Ersteren directer Betheiligung im Sommer 1876 gleichzeitig mit der Verbindung Wien-Berlin-Greenwich ausgeführt. Ich habe dann alsbald nach Empfang der Odessaer Beobachtungen die Bearbeitung vorgenommen und hierauf das sämmtliche grossentheils druckfertige Material, leider ohne Abschrift zu nehmen, Herrn Prof. Block zugestellt, damit derselbe zunächst untersuche, ob sich für die Aufklärung einer Differenz, die in den Uhrvergleichungen zurückgeblieben war und deren Ursprung ich in Odessa glaubte suchen $z u$ müssen, daselbst ein Anhalt finden lasse und hierauf die von ihm in Aussicht gestellte Publication ins Werk setze. Seitdem habe ich aus mir unbekannten Grlinden über das Schicksal der Arbeit nichts weiter zu erfahren vermocht. Bei der Bedeutung, welche eine directe Verbindung der beiden Sternwarten beanspruchen darf, will ich die wenigen auf das Resultat bezuglichen und von den definitiven Werthen nur unerheblich abweichenden Zahlen, die ich in meinen Papieren vorfinde, hier zusammenstellen. Dieselben sind, was die Uhrdifferenzen betrifft, nur ein einseitiges Resultat des ges ammten Beobachtungsmaterials, da die aus der Methode der Coincidenzbeobachtungen, welche neben dem gewöhnlichen Registrirverfahren zur Anwendung kam, erlangten Zahlen nicht mehr in meinen Händen sind. Zwischen den Resultaten beider Vergleichungsmethoden bestand ein erheblicher Unterschied (meiner Erinnerung nach etwa o.07), fur den ich vergeblich nach einer Erklärung gesucht habe; ich bin aber geneigt, den constanten Fehler, der sich darin ausspricht, eher dem Verfahren der Coincidenzbeobachtungen (Beobachtung der Coincidenz der Anschläge des Relais mit den Schlägen eines Halbsecunden-Chronometers bez. eines 130 Schlägers), als der bei der Anwendung des Registrirens auch bei Sterndurchgängen jedenfalls gleichartigeren Methode der gewöhnlichen Registrirsignale zuzuschreiben. Die Zeitbestimmungen wurden an beiden Stationen an den festen Meridianinstrumenten, in Berlin an dem grossen Pistor $\&$ Martins'schen, in Odessa an dem Repsold'schen Meridiankreise gemacht. Bei der Registrirung der Sterndurchgänge in Berlin wurde an der Mehrzahl der Abende das fur die Längenbestimmung mit Wien und Greenwich dienende Dosenrelais angewandt, wăhrend bei dem Signalaustausch mit Odessa ein mit dem dortigen ganz gleichartiges polarisirtes Relais benutzt werden musste; an denjenigen Abenden, wo eine Verbindung allein mit letzterer Station stattfand, sind auch die Fadenantritte mittelst des polarisirten Relais registrirt worden. Der Unterschied beider Relais wurde sorgfältig ermittelt. In der nachstehenden Tabelle bedarf nur die Corr.e uberschriebene Columne einer Erläuterung. Juli 26 war in Odessa beim Empfang der Berliner Signale die Einschaltung des Registrirapparates verabsäumt worden; an die Differenz der Uhrzeiten ist daher das Mittel der in der 3. Col. enthaltenen Stromverzögerungen angebracht. Juli 29 sind die Fadenantritte in Berlin ohne Einschaltung des Relais registrirt worden; die hieraus entstandene Verfruhung der Antrittssignale wurde zu $0 \$ 048$ ermittelt. Die bei den ubrigen Tagen angeführte Correction entspricht der relativen Verzögerung des Dosenrelais gegen das polarisirte Relais.

\begin{tabular}{|c|c|c|c|c|c|c|c|c|c|c|c|c|}
\hline 1876 & $\left|\begin{array}{c|}\text { Diff. der } \\
\text { Uhrzeiten }\end{array}\right|$ & $\begin{array}{c}\text { Einf.Strom- } \\
\text { verzög. }\end{array}$ & $\begin{array}{c}\Delta u \\
\text { (Odessa) }\end{array}$ & Lage & $* *$ & $\underset{\text { (Berlin) }}{\Delta u}$ & Lage & $* *$ & $\lambda_{0}$ & Corr. & $\lambda$ & $p$ \\
\hline Juli 26 & $\begin{array}{c}1^{h} 9^{m} \\
23^{3} 8_{4} 6\end{array}$ & - & $-20: 251$ & $\mathbf{W}$ & 20 & $-23^{5.812}$ & 0 & 21 & $\begin{array}{c}1^{\mathrm{h}} 9^{\mathrm{m}} \\
27^{\mathrm{s}} \cdot 407\end{array}$ & +0.058 & $\begin{array}{l}1^{h} 9^{m} \\
27^{3} \cdot 4^{6}\end{array}$ & 1.02 \\
\hline 27 & 26.189 & 0.047 & $-22.66_{3}$ & $\mathbf{W}$ & 22 & -24.036 & $\mathbf{W}$ & 20 & 562 & -0.008 & 55 & 1.05 \\
\hline 29 & 30.895 & 60 & -28.005 & $\mathbf{O}$ & 25 & -24.594 & 0 & 16 & 484 & $+0.04^{8}$ & 53 & 0.98 \\
\hline 31 & $35.8 \times 3$ & 64 & -33.299 & $\mathbf{W}$ & 16 & $-24.93 \mathrm{I}$ & O & I 9 & 445 & -0.008 & 44 & 0.87 \\
\hline Aug. 2 & 40.853 & 70 & $-3^{8.717}$ & $\mathrm{O}$ & 21 & $-25 \cdot 308$ & $\mathbf{W}$ & 25 & 444 & -0.008 & 44 & 1.14 \\
\hline $\begin{array}{l}7 \\
8\end{array}$ & $\begin{array}{l}54.152 \\
56.807\end{array}$ & $\begin{array}{l}78 \\
63\end{array}$ & $\begin{array}{r}-53.134 \\
-56.064\end{array}$ & $\begin{array}{l}\mathbf{W} \\
\mathbf{w}\end{array}$ & 23 & $\begin{array}{r}-26.405 \\
-26.622\end{array}$ & $\begin{array}{l}\mathbf{W} \\
\mathbf{O}\end{array}$ & 8 & $\begin{array}{l}423 \\
455\end{array}$ & $\begin{array}{c}-0.008 \\
-\end{array}$ & $\begin{array}{l}42 \\
45\end{array}$ & $\begin{array}{l}0.59 \\
0.43\end{array}$ \\
\hline 9 & 59.551 & 61 & -58.939 & O & 18 & -26.893 & 0 & 18 & 505 & - & 51 & 0.90 \\
\hline 10 & 61.990 & $6 I$ & -61.618 & $\mathbf{W}$ & 18 & -27.116 & $\mathbf{W}$ & 18 & 488 & 一 & 49 & $0.9^{\circ}$ \\
\hline 13 & 69. & 57 & -70.037 & O & 19 & -27.631 & $\mathbf{O}$ & 15 & 402 & -0.008 & 39 & 0.84 \\
\hline 14 & 72.470 & 48 & -72.865 & O & 21 & -27.852 & $\mathbf{W}$ & 16 & 457 & - & 46 & 0.91 \\
\hline 15 & 75.090 & $5^{2}$ & -75.648 & W & 8 & -28.059 & $\mathbf{W}$ & 12 & 501 & -0.008 & 49 & 0.48 \\
\hline 17 & 79.723 & 43 & -80.798 & W. & 23 & -28.522 & $\mathrm{O}$ & -I 9 & 447 & -0.008 & 44 & 1.04 \\
\hline 19 & $84 \cdot 34^{8}$ & 46 & $-85.93 \mathrm{I}$ & $\mathbf{W}$ & I I & -29.047 & $\mathbf{W}$ & $2 I$ & 464 & -0.008 & 46 & 0.72 \\
\hline
\end{tabular}

Lässt man bei der Ableitung des Mittelwerthes die in jedem Fall nur unbedeutenden Unterschiede in der Genauigkeit der Uhrdifferenzen unbeachtet - ausser Juli 26 ist nur am letzten Abend in Folge längerer Versuchsreihen die Anzahl der Signale erheblich hinter der normalen zurïckgeblieben (27 gegen 70) -, so erhält man im Mittel:

$$
\lambda=x^{h} 9^{m} 27^{s} \cdot 466 \text { w. F. } \pm 0 ! 008 \text {. }
$$


Berücksichtigt man die verschiedene Anzahl der Sterne, indem man als Gewicht $p=\frac{2}{20} \frac{a b}{(a+b)}$ einfuhrt, wo $a$ und $b$ die Anzahl der an beiden Stationen beobachteten Zeitsterne bezeichnen, so wird:

$$
\lambda=1^{\mathrm{h}} 9^{\mathrm{m}} 27^{5} \cdot 469 \text { Gew. } 11.87 \text { w. F. } \pm 0.008 \text {. }
$$

Endlich giebt die Trennung der einzelnen Lagen:

\begin{tabular}{|c|c|c|c|c|}
\hline Odessa & Berlin & $\lambda$ & Anz. & Gew. \\
\hline W & 0 & $1^{\mathrm{h}} 9^{\mathrm{m}_{2} 7^{\mathrm{s}} \cdot 44^{8}}$ & 4 & 3.36 \\
\hline $\mathbf{w}$ & $\mathbf{w}$ & 490 & 5 & 3.74 \\
\hline $\mathrm{O}$ & 0 & 480 & 3 & 2.72 \\
\hline 0 & w & 449 & 2 & 2.05 \\
\hline & & $\begin{array}{l}\text { I. } 927.467 \\
\text { w. F. } \pm 0.00\end{array}$ & & 11.26 \\
\hline
\end{tabular}

Ich nehme hiernach als wahrscheinlichsten Werth:

$$
\lambda=1^{\mathrm{h}} 9^{\mathrm{m}} 27^{\mathrm{s}}: 467 \text {. }
$$

Die persönliche Gleichung $\mathrm{Bl}-\mathrm{B}$ ist bei zweimaliger Anwesenheit des Herrn Prof. Block, zuerst im August 1875 und ein zweites Mal kurz vor Beginn der Längenbestimmung, theils am grossen Berliner Meridiankreis, theils am kleineren Pistor'schen Instrument bestimmt worden. Die einzelnen Tagesmittel aus den gemeinsain beobachteten Sternen sind (Block-Becker):

Grosser Meridiankreis.

$1875 \quad$ Anz.

Aug. $25 \quad-0.14 \quad 15$

\begin{tabular}{l}
$26 \quad-0.11 \quad 10$ \\
\hline
\end{tabular}

$\begin{array}{lll}27 & -0.13 \quad 13\end{array}$

- $28 \quad-0.13 \quad 16$

Mittel nach Gew. -0.129

- ohne, -0.127

Mittel -0.128

Grosser Meridiankreis.

$$
\begin{array}{cccc}
\multicolumn{2}{c}{1876} & & \text { Anz. } \\
\text { Juli } & 9 & -0.13 & 6 \\
1 & 10 & -0.17 & 12 \\
711 & -0.17 & 10
\end{array}
$$

\begin{tabular}{|c|c|c|}
\hline 187 & & \\
\hline Juli & 9 & -0.11 \\
\hline • & I I & -0.07 \\
\hline & 12 & -0.16 \\
\hline
\end{tabular}

Mittel nach Gew. -0.161

Kleiner Meridiankreis.

Mittel nach Gew. -0.143
Zieht man, da eine Abhängigkeit der Gleichung von den benutzten Instrumenten nicht hervortritt, die an demselben Tage an beiden Instrumenten erhaltenen IVerthe $\mathbf{z u}$ sammen, so ergiebt sich (Block-Becker):

$$
\begin{array}{rrr}
1876 & & \text { Anz. } \\
\text { Juli } 9 & -0.12 & 9 \\
10 & -0.17 & 12 \\
111 & -0.15 & 12 \\
12 & -0.16 & 14 \\
\text { Mittel nach Gew. }-0.152 & \\
\text { ohne, } & -0.150 & \\
\text { Mittel }-0.151 &
\end{array}
$$

Ausser diesen Vergleichungen liegt noch eine Bestimmung der persönlichen Gleichung aus gleichzeitigen Zeitbestimmungen ( 1876 Juli 9) beider Beobachter an beiden Instrumenten vor. Es wurde gefunden:

$\Delta u \mathrm{Bl}\left(\mathrm{Kl}\right.$.Mer.-Kr.) $-\Delta u \mathrm{~B}$ (Gr.Mer.-Kr.) $=+0^{5} \mathrm{r} 5$ ( 5 Sterne) $\Delta u \mathrm{Bl}(\mathrm{Gr} . M e r .-K r)-.\Delta u \mathrm{~B}(\mathrm{Kl} . M e r .-K r)=.+0.1 \mathrm{l}$ (6 Sterne)

woraus im Mittel, unabhängig von den Eigenthümlichkeiten der beiden Instrumente:

$$
\mathrm{Bl}-\mathrm{B}=-0.13
$$

folgt. - Vereinigt man die drei Werthe:

$$
\begin{array}{cc}
1875 & -0.128 \\
1876 & -0.151 \\
, & -0.130
\end{array}
$$

indem man dem ersten und dritten einfaches, dem mittleren doppeltes Gewicht zutheilt, so erhält man als definitiven Werth der persönlichen Gleichung:

$$
\mathrm{Bl}-\mathrm{B}=-0 ! 140 .
$$

Die gemeinsame Beobachtung eines Polsterns ( $\delta$ Urs. min.) an 4 Tagen $(1875)$ hat keinen zu verbürgenden Unterschied der Auffassung ergeben. Mit Berücksichtigung obiger Gleichung und des westlichen Abstandes der Mitte der Axe des grossen Meridiankreises in Berlin vom Centrum der grossen Kuppel (0.034) folgt endlich:

Mitte der Axe des Repsold'schen Meridiankreises in Odessa östlich vom Centrum der grossen Kuppel der Berliner Sternwarte :

$$
I^{\mathrm{h}} 9^{\mathrm{m}} 27^{\mathrm{s}} 29 \text {. }
$$

Gotha 1887 Nov. 9 .

E. Becker.

Berichtigung zu Nr. 2817 Bd. 118 S. 143 Z. 17 v. u. statt: schwächere lies: schwächende

\section{Inhalt:}

Zu Nr. 2820. $K$. Oertel. Vergleichung der in den ,Greenwich Observationse von 1877 bis 1884 enthaltenen Sternverzeichnisse mit den beiden Catalogen der Astronomischen Gesellschaft. 177. - E. Becker. Ceber die im Jahre 1876 ausgefülurte Längenbestimmung $2 w i s c h e n$ Berlin und Udessa. 189. - Berichtigung. 191. 